\title{
Ранняя диагностика метаболического синдрома на основе неинвазивной визуализации органов гепатопанкреатобилиарной системы
}

\author{
И.А. Шишова ${ }^{1,2}$, О.А. Ефремова ${ }^{1}$, П.Е. Чернобай ${ }^{1}$, Ш. Абдулгани ${ }^{1}$ \\ ${ }^{1}$ Белгородский государственный национальный исследовательский университет, \\ Россия, 308015, г. Белгород, ул. Победы, д. 85 \\ ${ }^{2}$ Белгородский онкологический диспансер, \\ Россия, 308010, г. Белгород, ул. Куйбышева, д. 1 \\ E-mail: shishova@bsu.edu.ru
}

\begin{abstract}
Аннотация. В статье представлены результаты исследования, проведенного с целью определения ранних значимых маркеров метаболического синдрома (MC) на основе неинвазивной визуализации внутренних органов брюшной полости. В исследование были включены 125 человек, 100 человек основной группы были поделены на две подгруппы. I подгруппа - пациенты с нормативными значениями индекса массы тела (ИМТ), но с повышенными показателями коэффициента окружность талии / объем бедер (ОТ/ОБ); II - с повышенными показателями ИМТ и ОТ/ОБ. Контрольная группа составила 25 человек. По данным анализа полученных результатов настоящего исследования была выявлена прямая зависимость между величиной ИМТ, показателем абдоминального ожирения (АО) (ОТ/ОБ) и изменением ультразвуковой эхоструктуры печени, поджелудочной железы, линейными размерами печени и показателем жесткости печеночной ткани. К ранним признакам МС были отнесены: 1. Повышение эхогенности и снижение звукопроводимости структуры печени.

2. Гепатомегалия (увеличение линейных размеров печени: краниовертикальный размер (КВР) (правой доли) более 150 мм, краниокаудальный размер (ККР) (левой доли) более 100 мм, хвостатой доли более 54 мм) была выявлена у 51 человека II подгруппы $(40,8 \%)(\mathrm{p}<0,05)$.

3. Изменение ультразвуковой структуры содержимого и стенки желчного пузыря, полипоз.

4. Повышение эхогенности ткани поджелудочной железы.
\end{abstract}

Ключевые слова: ультразвуковая диагностика, метаболический синдром, стеатогепатоз.

Для цитирования: Шишова И.А., Ефремова О.А., Чернобай П.Е., Абдулгани Ш. 2020. Ранняя диагностика метаболического синдрома на основе неинвазивной визуализации органов гепатопанкреатобилиарной системы. Актуальные проблемы медицины, 43 (3): 351-364. DOI: 10.18413/2687-0940-2020-43-3-351-364

\section{Early diagnostics of metabolic syndrome on the basis of non-invasive visualization of the hepatopancreatobiliary system organs}

\author{
Irina A. Shishova, ${ }^{1,2}$, Olga A. Efremova ${ }^{1}$, Pavel Ye. Chernobay ${ }^{1}$, Shakhnoza Abdulgani ${ }^{1}$ \\ ${ }^{1}$ Belgorod National Research University, \\ 85 Pobedy St., Belgorod, 308015, Russia \\ ${ }^{2}$ Belgorod Oncological Dispensary, \\ 1 Kuibysheva St., Belgorod, 308010, Russia \\ E-mail: shishova@bsu.edu.ru
}

\begin{abstract}
The article presents the results of a study conducted to identify early significant markers of metabolic syndrome (MS) based on non-invasive visualization of the internal organs of the abdominal cavity. The study included 125 people, 100 people of the main group were divided into two subgroups. Subgroup I patients with normative values of body mass index (BMI), but with increased indices of the waist
\end{abstract}


circumference / hip circumference; II - with increased BMI and waist circumference / hip circumference. The control group consisted of 25 people. According to the analysis of the obtained results of this study, a direct relationship was revealed between the BMI value, the indicator of abdominal obesity (waist circumference / hip circumference) and changes in the ultrasonic echostructure of the liver, pancreas, linear dimensions of the liver and the stiffness of the liver tissue. The early signs of MS included:

1. Increased echogenicity and decreased sound conductivity of the liver structure.

2. Hepatomegaly (an increase in the linear dimensions of the liver: craniovertical size (right lobe) more than $150 \mathrm{~mm}$, craniocaudal size (left lobe) more than $100 \mathrm{~mm}$, caudate lobe more than $54 \mathrm{~mm}$ ) was detected in 51 people of subgroup II $(40.8 \%)(\mathrm{p}<0.05)$.

3. Changes in the ultrasonic structure of the contents and wall of the gallbladder, polyposis.

4. Increasing the echogenicity of the pancreatic tissue.

Keywords: ultrasound diagnostics, metabolic syndrome, steatohepatosis.

For citation: Shishova I.A., Efremova O.A., Chernobay P.E., Abdulgany Sh. 2020. Early diagnostics of metabolic syndrome on the basis of non-invasive visualization of the hepatopancreatobiliary system organs. Challenges in Modern Medicine, 43 (3): 351-364 (in Russian). DOI: 10.18413/2687-0940-2020-43-3-351-364

\section{Введение}

В настоящее время вопрос раннего выявления компонентов метаболического синдрома (МC) прочно удерживает лидирующие позиции в медицинской литературе многих стран мира [Хайбуллина и др., 2014; Килина и др., 2015; Kimetal., 2018]. Особая значимость этой проблемы заключается в неуклонном росте числа заболевших, в том числе экономически активного возраста, и отсутствии условий для эффективной первичной профилактики и диагностики на доклинической фазе.

Российскими и зарубежными исследователями уже более 10 лет предпринимаются попытки систематизировать выявляемые в рамках рутинных инструментальных методов обследования пациентов с МС, изменение структуры печени, поджелудочной железы, толщины и структуры стенок артерий, а также выявить зависимость между патогенезом ремоделирования вышеуказанных органов-мишеней и патогенетическими факторами МС (гиперлипидемией, гиперинсулинемией, инсулинорезистентностью) [Хайбуллина и др., 2014; Килина и др., 2015; Kimetal., 2018].

В российской и международной медицинской литературе также активно дискутируется вопрос о механизме возникновения неалкогольной жировой болезни печени (НАЖБП). По мнению одних исследователей, первичным является отложение жировой ткани в ткани печени [Хайбуллина и др., 2014; Килина и др., 2015; Limetal., 2018].

Согласно приверженцам другой точки зрения, первичным является воспалительный процесс в гепатоцитах, вызывающий их повреждение и приводящий к развитию местных иммунно-воспалительных реакций, с активацией внутриклеточного перекисного окисления липидов и продукции коллагена [Тихонов и др., 1994].

Так как вопрос первичности ремоделирования структуры печени до сих пор остается дискутабельным, возникает проблема достоверной оценки возникающих на фоне МС изменений структуры печени. Вопрос диагностики ремоделирования структуры печени, несмотря на повышенный к нему интерес исследователей, достаточно сложен. Его сложность заключается как в отсутствии единых стандартов инструментальной диагностики диффузного поражения печени на фоне МС, так и ввиду ограничения распознавания плотности печеночной ткани в нативных инструментальных исследованиях [Хайбуллина и др., 2014; Килина и др., 2015; Kimetal., 2018; Limetal., 2018].

В рамках ультразвуковой диагностики НАЖБП не имеет четких критериев и в значительной степени операторозависима, что затрудняет установление этого диагноза в рамках рутинного ультразвукового обследования. Эффективным помощником при этом может стать эластографическая методика сдвиговой волны. С ее помощью возможно определение 
коэффициента жесткости ткани печени, его оценке по шкале Metavir и определения степени развития фиброзных изменений в структуре печени как показателя развития диффузного иммунно-воспалительного поражения и его исхода в диффузный или очаговый фиброз [Недогода и др., 2014; Килина и др., 2015; Ikuraetal., 2014; Limetal., 2018].

НАЖБП в современной литературе рассматривается не только как нозологическая форма, от которой зависит риск кардиоваскулярных катастроф и степень прогрессирования сердечно-сосудистых заболеваний, хронической болезни почек и сахарного диабета 2 типа, но и как независимый фактор риска и ранний маркер МC [Кособян и др., 2010; Käräjämäkietal., 2017], хотя на сегодняшний день конкретных, значимых показателей, характеризующих развитие МС по данным ультразвукового исследования внутренних органов брюшной полости, нет.

Это свидетельствует о высокой актуальности вопроса раннего выявления МС неинвазивными методиками.

Цель исследования: выделить наиболее ранние значимые маркеры МС на основе неинвазивной визуализации внутренних органов брюшной полости.

\section{Материалы и методы исследования}

В исследование были включены 125 человек, жителей города Белгорода и Белгородской области. Основную исследуемую группу составили 100 человек, которые были разделены на 2 подгруппы: 1 - пациенты, имеющие нормальный ИМТ, но повышенный коэффициент ОТ/ОБ, численностью 38 человек, 2 - пациенты, имеющие повышенный ИМТ, ОТ/ОБ и клинико-лабораторные критерии МС, численностью 62 человек. 25 человек не имели клинико-лабораторных признаков МС, они образовали группу контроля (КГ).

Все группы были сопоставимы по полу и возрасту: в первую подгруппу были включены 6 мужчин (15,79\%) и 32 женщины $(84,2 \%)$, во вторую - 15 мужчин $(24,19 \%)$ и 47 женщин (75,81 \%). В группу контроля -12 мужчин (48 \%) и 13 женщин $(52 \%)(\mathrm{p}<0,05)$.

Критериями отбора пациентов в исследование являлось наличие двух и более признаков: АО, наследственный анамнез и наблюдение у профильных специалистов по поводу артериальной гипертензии (с ежесуточными цифрами артериального давления (АД) более 130/90 мм. рт. ст.), нарушение толерантности к глюкозе или сахарного диабета 2 типа, повышение уровня холестерина липопротеидов низкой плотности сыворотки крови более 3,0 ммоль/л, повышение триглицеридов сыворотки крови более 1,69 ммоль/л, снижение холестерина липопротеидов высокой плотности в сыворотке крови: мужчины - менее 40 мг/дл (менее 1,04 ммоль/л), женщины - менее 50 мг/дл (менее 1,29 ммоль/л), повышение глюкозы сыворотки крови натощак (более 6,1 ммоль/л).

Лабораторно-клинические показатели уровня общего холестерина, ЛПНП, триглицеридов и глюкозы крови определялись в клинической лаборатории Белгородского онкологического диспансера, данные были получены из медицинской документации во время проведения общего обследования пациента во время его стационарного лечения.

В качестве основной инструментальной методики была использована неинвазивная трансабдоминальная ультразвуковая сонографическая методика обследования органов брюшной полости.

В качестве ультразвукового прибора была использована система экспертного класca Logic E 9 фирмы «General Electric» и Esaote MyLab Twice. Ультразвуковое исследование выполнялось натощак, с использованием широкополосного конвексного датчика с частотой 3,5 МГц и линейного датчика с частотой 7,5 МГц.

В качестве используемых методик осмотра печени, поджелудочной железы, желчного пузыря были использованы стандартные, предложенные Митьковым В.В. протоколы обследования [Митьков, 2011].

Во время исследования проводилась оценка расположения, формы, строения, линейных размеров и структуры (однородность, эхогенность, звукопроводимость) печени, 
поджелудочной железы, толщины стенки желчного пузыря, наличия в просвете внутрипросветных пристеночных образований, конкрементов или осадка. За нормативы измерений и критерии оценки состояния печени в рамках использованного в работе ультразвукового метода были приняты стандартные, предложенные Митьковым В.В. [Митьков, 2011].

\section{Результаты исследования}

В результате проведенного обследования были получены следующие данные (представлены в таблице 1).

Таблица 1

Table 1

Данные обследования пациентов I и II подгруппы основной группы (ОГ)

Survey data of patients of I and II subgroups of the main group (MG)

\begin{tabular}{|l|c|c|c|c|c|c|c|c|}
\hline \multicolumn{1}{|c|}{ Параметры } & \multicolumn{4}{|c|}{ Подгруппа I $(\mathrm{n}=38)}$. & \multicolumn{4}{c|}{ Подгруппа II (n =66). } \\
\hline & mean & $\min$ & $\max$ & Std. Dev. & mean & $\min$ & $\max$ & Std. Dev. \\
\hline Возраст & 54,2 & 40 & 62 & 6,19 & 54,87 & 43 & 68 & 6,41 \\
\hline ИМТ & 28,7 & 26 & 30 & 1,01 & 33,8 & 31 & 37,6 & $1,71^{*}$ \\
\hline ОТ/ОБ & 1.03 & 0,8 & 1,25 & 0,10 & 1,1 & 0,92 & 1,34 & 0,10 \\
\hline КВР & 145 & 138 & 151 & 4,16 & 166,5 & 144 & 205 & $15,70^{*}$ \\
\hline Индекс жесткости & 5,5 & 4,2 & 6,4 & 0,59 & 7,2 & 5,4 & 8,68 & $0,62^{*}$ \\
\hline
\end{tabular}

Примечание: * - достоверность данных между группами, $\mathrm{p}<0,05$.

У пациентов обеих подгрупп ОГ были выявлены изменения эхоструктуры паренхимы печени, выражающиеся в снижении звукопроводимости, ослаблении выраженности сосудистого рисунка по периферии долей печени (в режиме ЦДК), а также повышении эхогенности (эхоплотности) печени (рис. 1, 2).

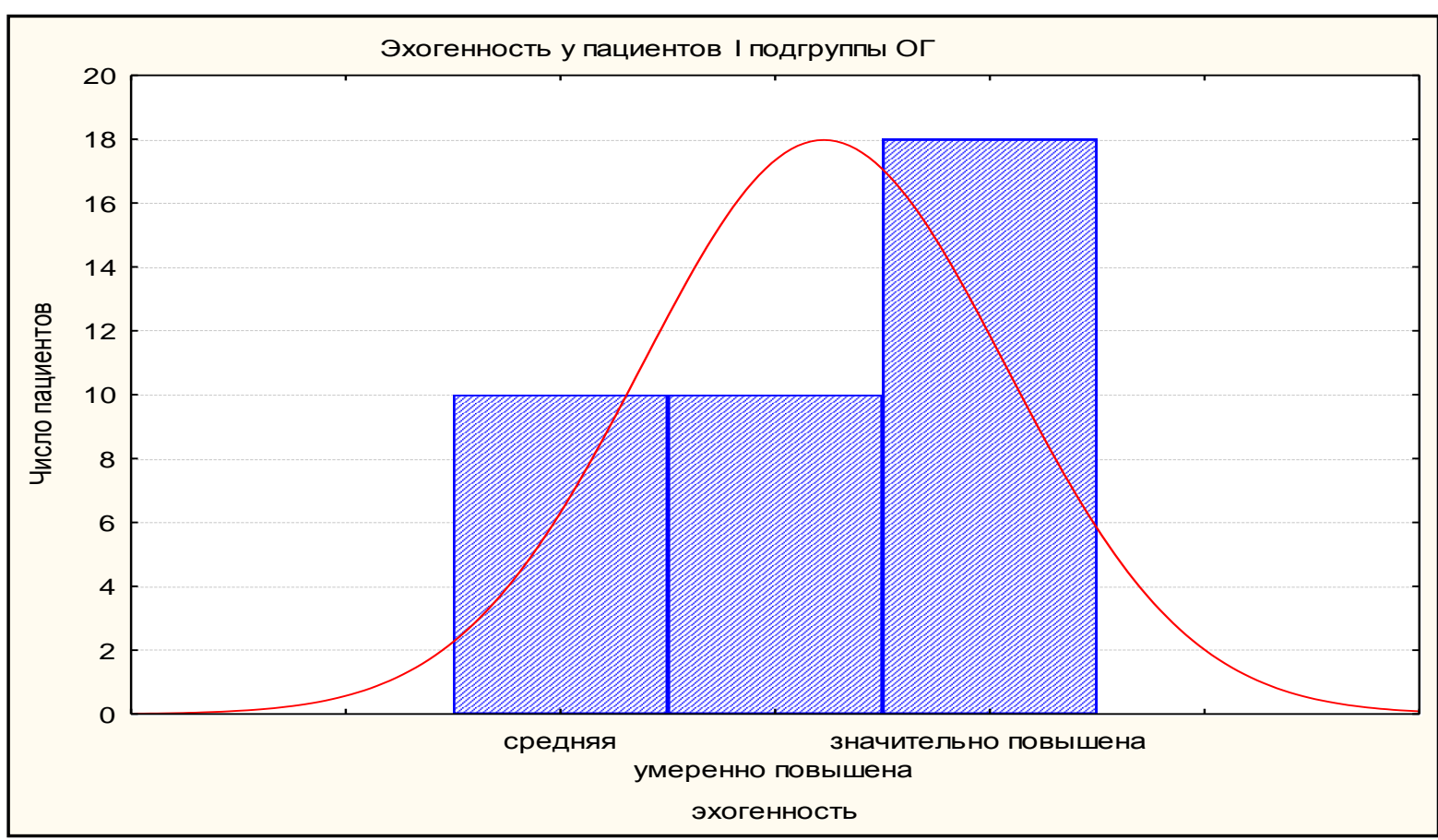

Рис. 1. Эхогенность печени у пациентов I подгруппы

Fig. 1. Echogenicity of the liver in patients of subgroup I

У 18 пациентов $(47,37$ \%) из I подгруппы эхогенность печени была повышена значительно, у 10 (26,3\%) - умеренно, у 10 (26,3\%) - повышения эхогенности печени не определялось. 


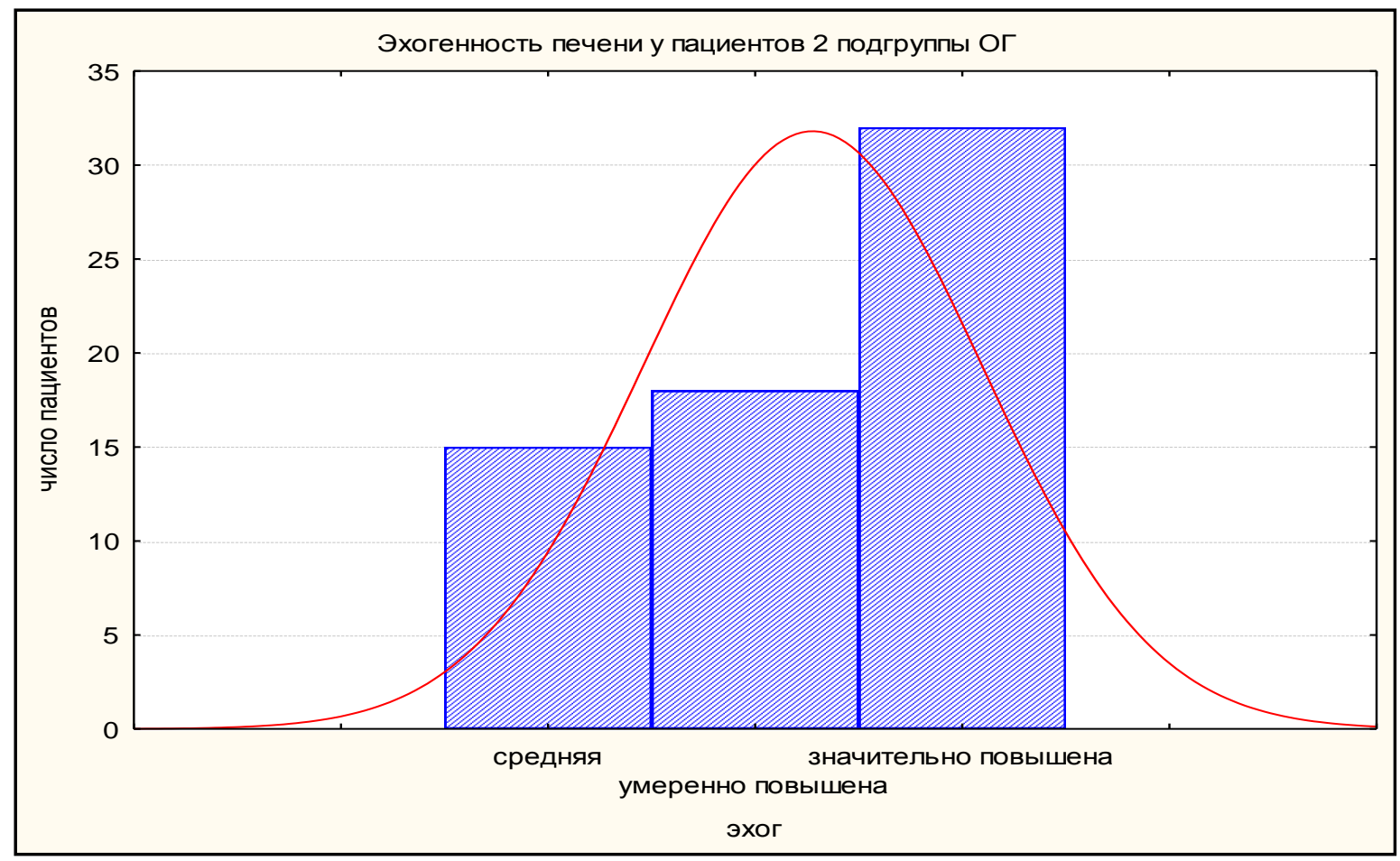

Рис. 2. Эхогенность печени у пациентов II подгруппы

Fig. 2. Echogenicity of the liver in patients of subgroup II

Bo II подгруппе у 33 пациентов (53,23 \%) эхогенность печени была повышена значительно, у 16 пациентов (25,81 \%) - умеренно повышена, у 15 (20,97 \%) пациентов - повышения эхогенности не отмечалось. В общем, у пациентов ОГ повышение эхогенности отмечалось у 77 человек (77\% от всего числа пациентов ОГ). Причем выраженность изменения эхогенности печени определялась в прямой зависимости от ИМТ (рис. 3). В наибольшей степени эта зависимость определялась у пациентов II подгруппы ОГ, имеющих повышенные ИМТ и ОТ/ОБ (рис. 4).

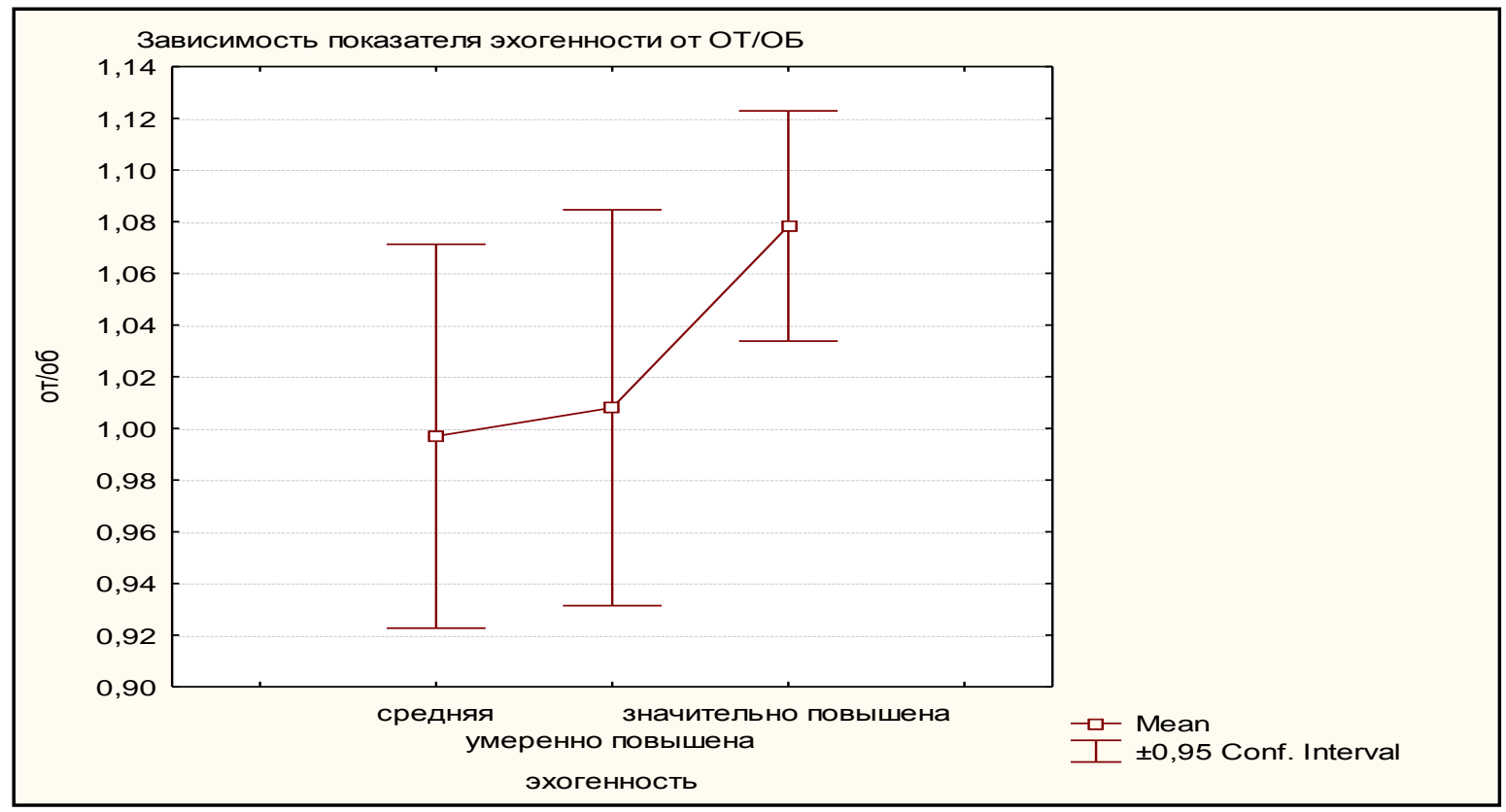

Рис. 3. Зависимость эхогенности печени от показателя ОТ/ОБ у пациентов I подгруппы

Fig. 3. Dependence of liver echogenicity on the OT / OB index in patients of subgroup I 


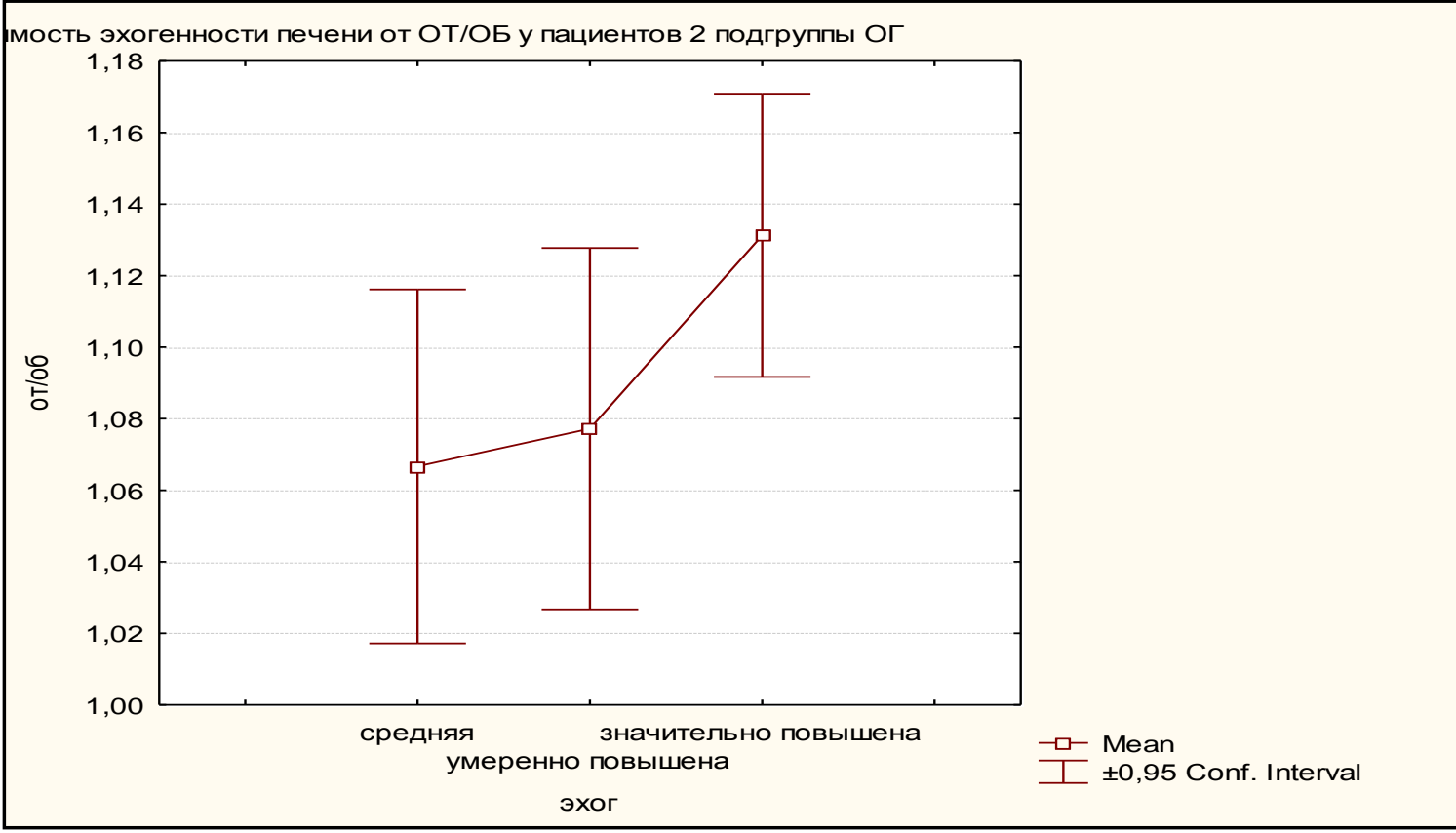

Рис. 4. Зависимость эхогенности печени от показателя ОТ/ОБ у пациентов II подгруппы

Fig. 4. Dependence of liver echogenicity on the OT / OB index in patients of subgroup II

Таким образом, в первой подгруппе была выявлена корреляция средней силы между уровнем показателя эхогенности и ОТ/ОБ $(\mathrm{r}=0,6)$, во второй подгруппе по этим же показателям определялась высокая корреляция $(\mathrm{r}=0,8)$.

Схожая тенденция определялась и по показателю звукопроводимости печеночной ткани у пациентов обеих групп (рис. 5, 6).

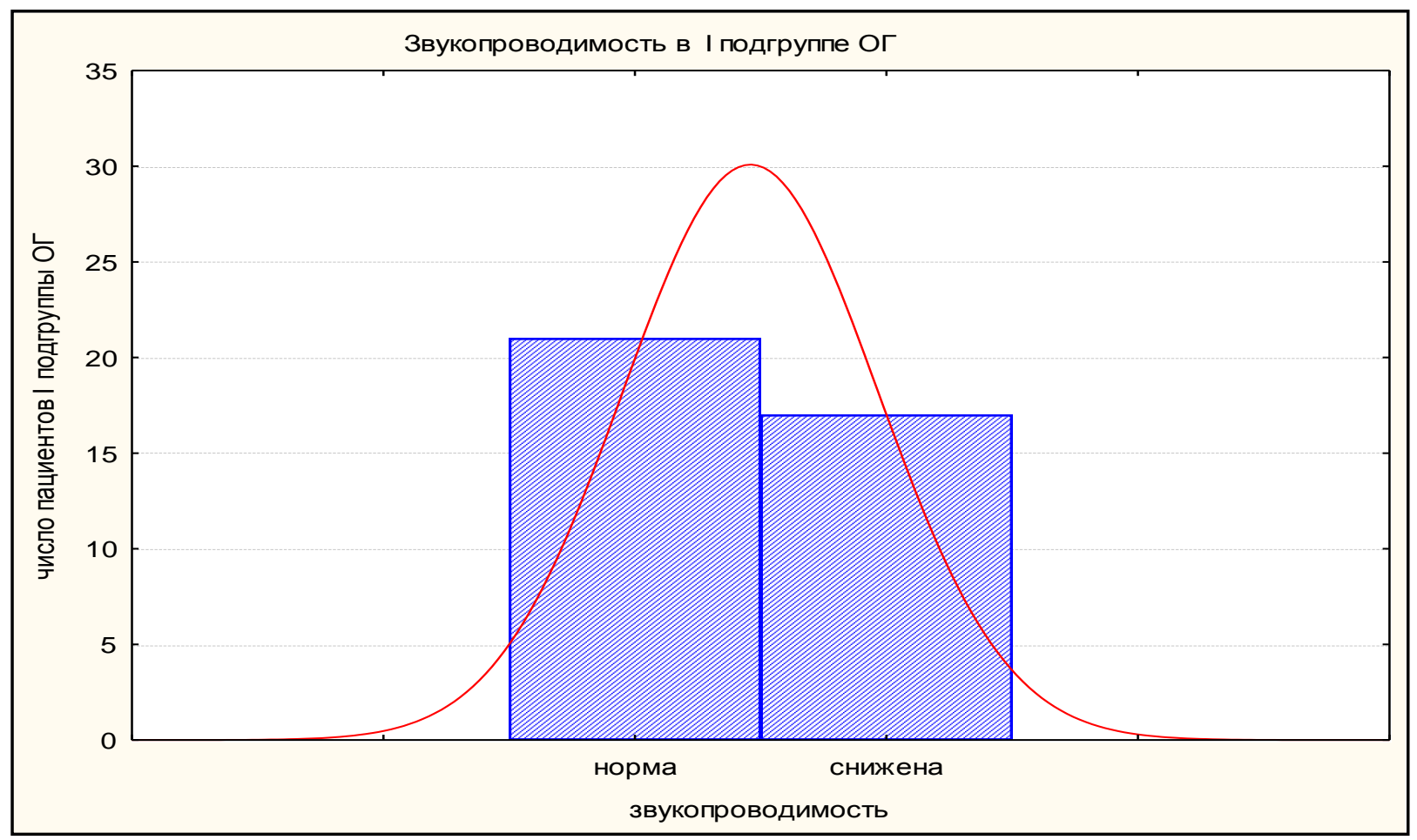

Рис. 5. Звукопроводимость у пациентов I подгруппы

Fig. 5. Sound conductivity in patients of subgroup I 


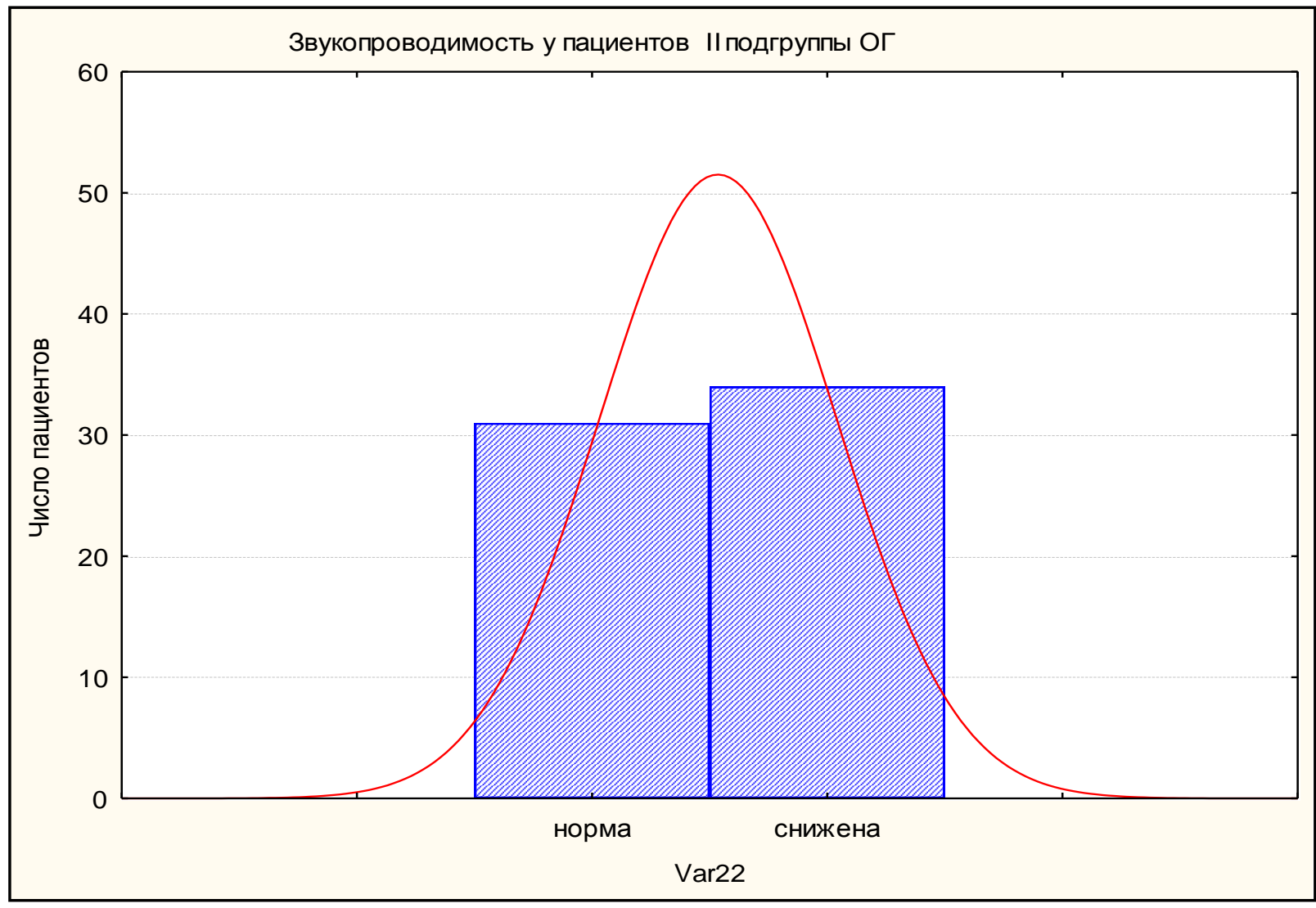

Рис. 6. Звукопроводимость у пациентов II подгруппы

Fig. 6. Sound conductivity in patients of subgroup II

(II подгруппа - корреляция звукопроводимости и ОТ/ОБ $\mathrm{r}=0,7$ )

Снижение звукопроводимости отмечалось у 17 пациентов I подгруппы (44,7 \%) и 34 пациентов II подгруппы (54,87 \%), т. е. у 51 \% всех пациентов ОГ. У пациентов КГ изменения эхогенности и звукопроводимости выявлено не было.

Таким образом, в первой подгруппе была выявлена слабая корреляция между уровнем показателя звукопроводимости и ОТ/ОБ $(\mathrm{r}=0,4)$, во второй подгруппе по этим же показателям определялась корреляция средней силы $(\mathrm{r}=0,7)$.

Также у всех пациентов ОГ определялись изменения показателей линейных размеров долей печени, зависящие от коэффициентов ИМТ и ОТ/ОБ. У пациентов I подгруппы ОГ (без повышения ИМТ и абдоминальным ожирением) на фоне ультразвуковых признаков диффузного изменения структуры печени не была выявлена гепатомегалия (медиана КВР (правая доля) составила 145 мм (std.Dev. 9,17), максимальный размер составил 151 мм). У пациентов II подгруппы (имеющих клинико-лабораторные критерии МС и повышение ИМТ и ОТ/ОБ), у 8 пациентов (12,9 \%) показатели КВР соответствовали возрастному нормативу, у 57 пациентов $(91,94 \%)$ определялась гепатомегалия (медиана КВР составила 166,5 мм, максимальный размер - 205 мм). По всем пациентам ОГ наибольший разброс увеличения КВР приходится на показатель ИМТ

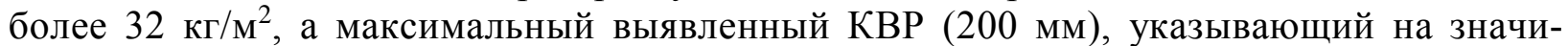

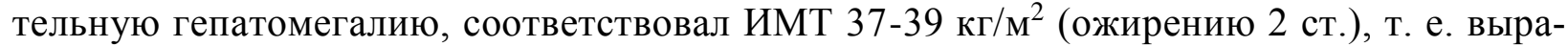
женность гепатомегалии зависит от ИМТ.

Динамика увеличения линейных размеров печени в зависимости от выраженности АО и ИМТ пациентов обеих подгрупп ОГ и КГ представлена в таблице 2. 
Размеры долей печени у пациентов ОГ и КГ

Sizes of liver lobes in patients with MG and CG

\begin{tabular}{|l|c|c|c|c|c|c|c|c|c|c|c|c|}
\hline & \multicolumn{4}{|c|}{ І подгруппа ОГ } & \multicolumn{3}{c|}{ ІІ подгруппа ОГ } & \multicolumn{4}{c|}{ КГ } \\
\hline & $\begin{array}{c}\text { mea } \\
\mathrm{n}\end{array}$ & $\min$ & $\max$ & $\begin{array}{c}\text { Std. } \\
\text { Dev }\end{array}$ & $\operatorname{mean}$ & $\min$ & $\max$ & $\begin{array}{c}\text { Std. } \\
\text { Dev }\end{array}$ & mean & $\min$ & $\max$ & $\begin{array}{c}\text { Std. } \\
\text { Dev. }\end{array}$ \\
\hline КВР & 145 & 138 & 151 & 9,17 & 166,5 & 144 & 205 & $15,71^{*}$ & 135,9 & 116 & 147 & $8,18^{*}$ \\
\hline ККР & 97,9 & 82 & 102 & 7,86 & 98,5 & 78 & 123 & 10,93 & 86,24 & 68 & 98 & $6,77^{*}$ \\
\hline $\begin{array}{l}\text { Хвостатая до- } \\
\text { ля }\end{array}$ & 59,6 & 43 & 95 & 11,83 & 64,6 & 43 & 98 & 15,01 & 53,7 & 43 & 74 & $6,25^{*}$ \\
\hline
\end{tabular}

Примечание: *- достоверность данных между группами, $\mathrm{p}<0,05$.

Наибольшие размеры долей соответствуют II подгруппе ОГ. Причем наиболее выражены колебания превышения линейных размеров в ОГ по параметру КВР (правая доля) [Abenavoli et al., 2016].

Аналогичная прямая зависимость прослеживается и между линейными размерами долей печени и показателем выраженности АО (ОТ/ОБ), а также и от ИМТ у пациентов II подгруппы ОГ, что можно расценить как раннее проявление ремоделирования печени на фоне МС (рис. 7).

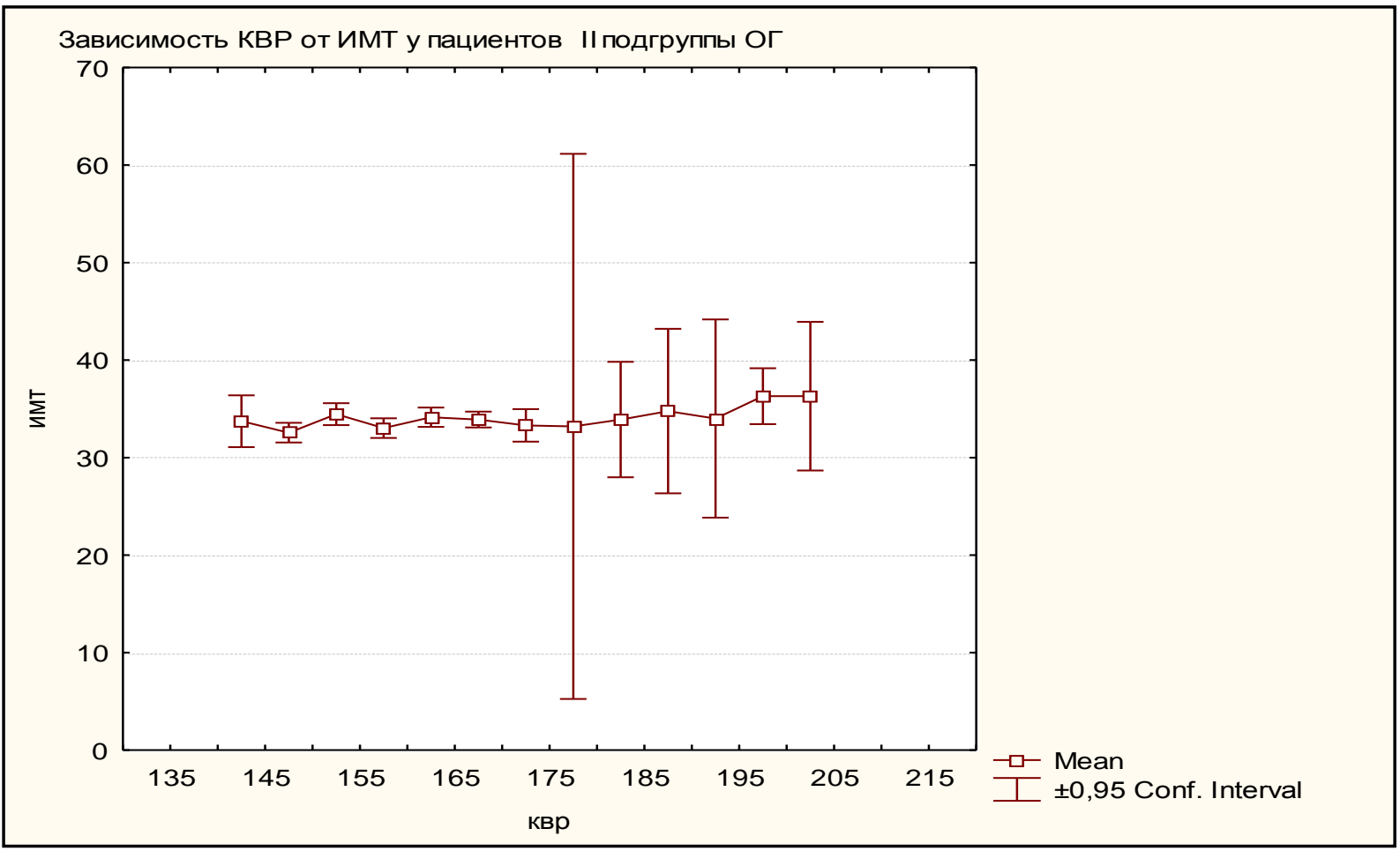

Рис. 7. Зависимость показателя КВР печени от ИМТ у пациентов II подгруппы

Fig. 7. Dependence of the liver CWR index on BMI in patients of subgroup II

У пациентов второй подгруппы была выявлена слабая корреляция между уровнем показателя КВР и ИМТ $(\mathrm{r}=0,4)$. Наибольшему показателю значения ОТ/ОБ, как показателю выраженности АО у пациентов с МС (II подгруппы ОГ), соответствуют увеличенные линейные размеры долей печени (гепатомегалии как важного проявления НАЖБП на фоне $\mathrm{MC}$ ).Также имеется аналогичная зависимость между изменением эхоструктуры пе- 
чени и поджелудочной железы, стенок желчного пузыря, наличием у пациентов желчекаменной болезни (ЖКБ) или полипоза от показателей ИМТ и коэффициента ОТ/ОБ, указывающая на связь поражения органов гепатобилиарной системы (как органов-мишеней) с МС. Причем чем выше показатели ИМТ, ОТ/ОБ, тем более выражены ультразвуковые изменения эхоструктуры печени и тем большая диагностическая точность НАЖБП (табл. 3).

Таблица 3

Table 3

Изменение ультразвуковой структуры печени, поджелудочной железы среди пациентов у пациентов ОГ и КГ

Changes in the ultrasound structure of the liver, pancreas among patients in patients with $\mathrm{MG}$ and CG

\begin{tabular}{|c|c|c|c|c|c|}
\hline & $\begin{array}{c}\text { Гепатомегалия, } \\
\text { панкреатомегалия, У3 } \\
\text { картина } \\
\text { диффузых изменений } \\
\text { структуры печени } \\
\text { (повышение } \\
\text { эхогенности, снижение } \\
\text { звукопроводимости, } \\
\text { ослабление } \\
\text { сосудистого рисунка) } \\
\text { и поджелудочной } \\
\text { железы, \% от общего } \\
\text { числа пациентов } \\
\text { (n = 125) } \\
\end{array}$ & $\begin{array}{c}\text { У3 картина } \\
\text { очаговых } \\
\text { изменений } \\
\text { структуры } \\
\text { поджелудочной } \\
\text { железы, без } \\
\text { изменения } \\
\text { структуры } \\
\text { печени, } \\
\text { увеличения их } \\
\text { размеров } \\
\text { (n=125) }\end{array}$ & $\begin{array}{c}\text { УЗ картина } \\
\text { нормальной } \\
\text { ультразвуковой } \\
\text { структуры } \\
\text { печени и } \\
\text { поджелудочной } \\
\text { железы без } \\
\text { увеличения их } \\
\text { размеров } \\
\text { (n=125) }\end{array}$ & $\begin{array}{c}\text { ЖКБ или } \\
\text { холецистэктомия } \\
\text { в анамнезе } \\
(\mathrm{n}=125)\end{array}$ & $\begin{array}{c}\text { Осадок в } \\
\text { просвете } \\
\text { ж/пузыря, } \\
(\mathrm{n}=125)\end{array}$ \\
\hline $\begin{array}{l}\mathrm{I} \text { І } . \\
\text { ОГ }\end{array}$ & 0 & 28 чел $(22,4 \%)$ & 10 чел (8\%) & $\begin{array}{c}\text { ЖКБ } 9 \text { чел } \\
(7,2 \%) \\
\text { ХЭ-4 чел } \\
(3,2 \%) \\
\end{array}$ & $\begin{array}{c}\text { осадок - } 24 \text { чел } \\
(19,2 \%), \text { поли- } \\
\text { поз - 5 чел. } \\
(4 \%) \\
\end{array}$ \\
\hline $\begin{array}{l}\text { II п. } \\
\text { ОГ }\end{array}$ & 51 чел. $(40,8 \%)^{*}$ & $\begin{array}{c}11 \text { чел. } \\
(8,8 \%)^{*}\end{array}$ & $0 *$ & $\begin{array}{c}\text { ЖКБ } 18 \text { чел. } \\
(14,4 \%), * \\
\text { XЭ } 5 \text { чел. (4 \%) }\end{array}$ & $\begin{array}{c}\text { осадок }-45 \text { чел } \\
(36 \%), * \\
\text { полипо }-8 \text { чел. } \\
(6,4 \%)\end{array}$ \\
\hline $\mathrm{K} \Gamma$ & 0 & 0 & 25 чел $(20 \%)$ & 0 & 0 \\
\hline
\end{tabular}

Примечание: * - достоверность данных между группами, $\mathrm{p}<0,05$.

Также были получены отличные от нормативных показатели индекса жесткости печеночной ткани по данным эластографии сдвиговой волны. У пациентов I подгруппы так же, как и у пациентов КГ, показатели жесткости печени соответствовали нормативным (медиана составила в I подгруппе ОГ 5.5 кПа, разброс коэффициента от 4.2 кПа до 6.4 кПа). У пациентов II подгруппы, имеющих клинико-лабораторные проявления МС, повышенные ИМТ и ОТ/ОБ, определялась более высокая медиана индекса жесткости печеночной ткани (7,2 кПа, разброс коэффициента - от 5,43 кПа до 8,68 кПа), что, согласно шкале Metavir, соответствует F1-F2 (начальным и умеренным фиброзным изменениям) по сравнению с пациентами КГ (медиана 3,89 кПа, разброс значений составил 2,6-5,2 кПа, что соответствует возрастным нормативам). Причем наибольшая выраженность фиброза (F2, 7,3-9,5 кПа по шкале Metavir) определялась у $58 \%$ пациентов II подгруппы ОГ

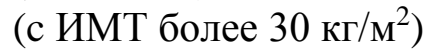

По показателю жесткости у пациентов в ОГ также определяется прямая зависимость его от уровня ИМТ и показателя ОТ/ОБ, что говорит о зависимости степени выраженности НАЖБП (в том числе и степени выраженности фиброзных изменений ткани печени как показателя исхода стеатогепатоза и стеатогепатита) от степени выраженности у 
пациента общего и абдоминального ожирения и массы тела (рис. 8, 9). По показателю жесткости печеночной ткани у пациентов КГтакая зависимость не определялась.

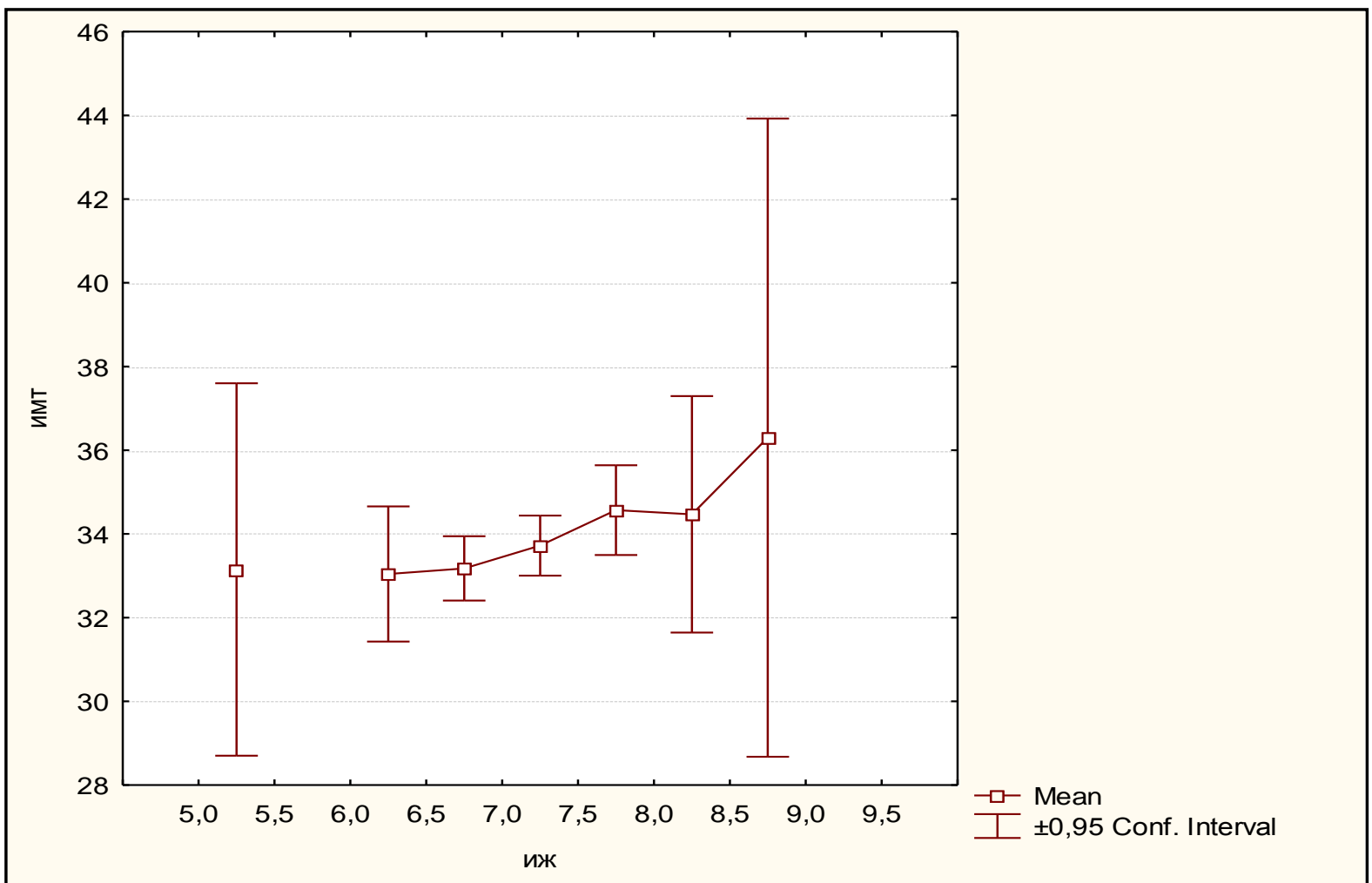

Рис. 8. Зависимость индекса жесткости печеночной ткани от показателя ИМТ

Fig. 8. Dependence of the stiffness index of the liver tissue on the BMI indicator

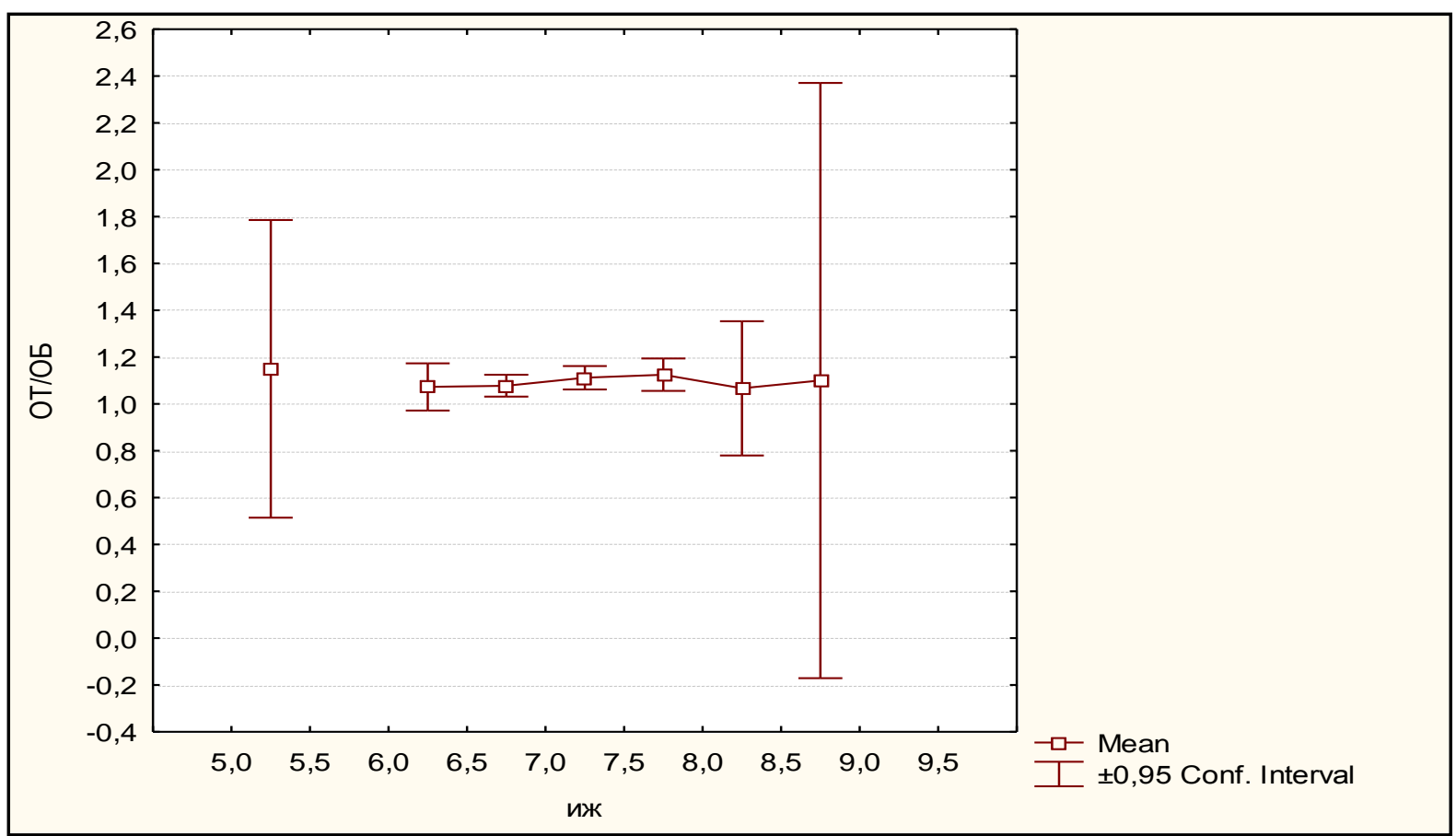

Рис. 9. Зависимость индекса жесткости печеночной ткани от показателя выраженности АО (ОТ/ОБ) Fig. 9. Dependence of the stiffness index of the liver tissue on the indicator of the severity $\mathrm{Of} \mathrm{AO}(\mathrm{OT} / \mathrm{OB})$ 
Во второй подгруппе была выявлена слабая корреляция между уровнем показателя индекса жесткости и ИМТ ( $\mathrm{r}=0,4)$, по показателям индекса жесткости и ОТ ОБ определялась корреляция средней силы $(\mathrm{r}=0,4)$.

Полученные данные коррелируют с данными, полученными в ходе исследований других авторов. Например, по данным Чесноковой Л.В. и соавт., в проведенном исследовании с применением стандартных ультразвуковых трансабдоминальных методик обследования печени и методики эластографии, признаки НАЖБП были выявлены у 89 \% пациентов с абдоминальным ожирением, у 100 \% пациентов с абдоминальным ожирением и клинико-лабораторными проявлениями $\mathrm{MC}$, а также из них у 46 \% были выявлены признаки фиброза в степени F0-F1 по шкале Metavir [Чеснокова и др., 2014].

Наличие сочетания выделенных ультразвуковых признаков ремоделирования печени, поджелудочной железы, стенки и содержимого желчного пузыря у одного и того же пациента. Данный признак был выявлен у 73 \% пациентов I подгруппы, 79,03 \% пациентов II подгруппы и не определялся у пациентов группы контроля.

\section{Обсуждение результатов}

Изучение МС имеет большое клиническое значение, поскольку, с одной стороны, это состояние является обратимым, т. е. при соответствующем лечении возможно исчезновение или снижение выраженности проявлений компонентов данного патологического состояния у пациентов, с другой - при отсутствии лечения резко возрастает риск развития таких социально значимых заболеваний, как сахарный диабет 2 типа и сердечнососудистая патология.

Печень как орган, активно участвующий в метаболических реакциях, выступает и как орган-мишень, и как основной фактор, провоцирующий и поддерживающий нарушения липидного и углеродного обмена, опосредованно влияя на инсулинорезистентность [Кособян и др., 2010; Чеснокова и др., 2014].

По данным анализа полученных результатов настоящего исследования была выявлена прямая зависимость между величиной ИМТ и показателя АО (ОТ/ОБ) и ультразвуковой эхоструктурой и линейными размерами печени, показателями жесткости печеночной ткани.

\section{Выводы}

У пациентов с факторами риска развития МС при ультразвуковом исследовании можно выделить ранние признаки МС. Эти признаки встречаются и у пациентов II подгруппы (имеющих повышенный ИМТ, выраженное АО (с повышением ОТ/ОБ)), но выраженные в большей мере. К таким ранним ультразвуковым признакам МС можно отнести:

1. Характерное изменение ультразвуковой структуры печени с повышением эхогенности, снижением звукопроводимости, обеднением сосудистого рисунка в режиме ЦДК - было выявлено у 28 пациентов (73,7 \%) I подгруппы и 51 пациентов (82 \%) II подгруппы $(\mathrm{p}<0,05)$.

2. Гепатомегалия (увеличение линейных размеров печени: краниовертикальный размер (КВР) (правой доли) более 150 мм, краниокаудальный размер (ККР) (левой доли) более 100 мм, хвостатой доли более 54 мм) - была выявлена у 51 человека II подгруппы $(40,8 \%)(\mathrm{p}<0,05)$.

3. Изменение ультразвуковой структуры содержимого желчного пузыря (в виде «густого» осадка, микролитов по типу «песка» или конкрементов в просвета желчного пузыря или протоках), полипоза и изменения эхоструктуры стенки желчного пузыря в виде ее утолщения, уплотнения (повышения эхогенности) и включений кальцинатов было выявлено у 42 пациентов I подгруппы (33,6 \%) и 76 пациентов II подгруппы $(60,8$ \%) (p < 0,05). 
4. Повышение эхогенности и звукопроводимости ткани поджелудочной железы отмечено у 28 человек (73,7 \%) I подгруппы и 51 пациентов (82 \%) II подгруппы (p < 0,05).

Таким образом, неинвазивная ультразвуковая визуализация позволяет на ранних этапах диагностировать ремоделирование органов мишеней $\mathrm{MC}$, а также проводить диагностику изменений их ультразвуковой структуры в динамике на фоне проводимой медикаментозной терапии.

\section{Список литературы}

1. Денисов Н.Л., Гриневич В.Б., Чернецова Е.В., Корноухова Л.А., Вострикова Е.Б., Чуприна С.В., Потапова И.В. 2017. Современные концепции патогенеза атеросклеротического поражения сосудистой стенки у пациентов с абдоминальным ожирением и различными формами неалкогольной жировой болезни печени. Вестник Национального медико-хирургического Центра им. Н.И. Пирогова. 1: 57-62.

2. Деомидова В.Н., Петрова О.В. 2013. Сравнительный анализ результатов эластографии сдвиговой волной и транзиентной эластографии в диагностике диффузных заболеваний печени // Ультразвуковая и функциональная диагностика. 5: 17-22.

3. Килина О.Ю., Ханарин Н.В., Иванова С.Н., Россова Н.А. 2015. Диагностические возможности ультразвукового исследования с оценкой гепаторенального индекса в диагностике неалкогольной жировой болезни печени у больных метаболическим синдромом. Вестник ХГУ им. Н.Ф. Катанова. 12: 56-59.

4. Кособян Е.П., Смирнова О.М. 2010. Современные концепции патогенеза неалкогольной жировой болезни печени. Сахарный диабет. 1: 55-63.

5. Лелюк В.Г., Лелюк С.Э. 2003. Ультразвуковая ангиология. 2-е издание, доп и перер. Москва. 336 с.

6. Митьков В.В. 2011. Практическое руководство по ультразвуковой диагностике. Общая ультразвуковая диагностика. Москва. Видар. 720 с.

7. Недогода С.В., Санина Т.Н., Чаляби Т.А., Барыкина И.Н., Цома В.В., Чумачек Е.В., Саласюк А.С., Хрипаева В.Ю., Смирнова В.О. 2014. Диагностика и особенности лечения неалкогольной жировой болезни печени у пациентов с метаболическим синдромом: возможности кардиологических и сахароснижающих препаратов. МС. 11: 66-69.

8. Тихонов А.И., Хворостинка В.Н., Ефремова О.А., Ярных Т.Г., Пушкарь С.Н., Котенко А.М. 1994. Средство, обладающее антиоксиоксидантным и гепатозащитным свойствами. Патент на изобретение RU 2020946 C1, 15.10.1994. Заявка № 4864094/14 от 05.09.1990.

9. Хайбуллина 3.Р., Косникова И.В. 2014. Проявления метаболического синдрома у больных мультифокальным атеросклерозом. Вестник НГПУ. 5 (21): 127-133.

10. Чеснокова Л.В., Петров И.М., Трошина И.А., Медведева И.В. 2014. Инсулинорезистентность, атерогенные нарушения и фиброз печени у больных с метаболическим синдромом. Ожирение и метаболизм. 2: 17-23.

11. Abenavoli L., Milic N., Di Renzo L., Preveden T., Medić-Stojanoska M., De Lorenzo A. 2016. Metabolic aspects of adult patients with nonalcoholic fatty liver disease. World. J. Gastroenterol. Aug 21; 22 (31): 7006-16.

12. Bhandarkar N.S., Brown L., Panchal S.K. 2019. Chlorogenic acid attenuates highcarbohydrate, high-fat diet-induced cardiovascular, liver, and metabolic changes in rats. Nutrition research. 62: 78-88.

13. Browning J.D., Kumar K.S., Saboorian M.H., Thiele D.L. 2004. Ethnic differences in the prevalence of cryptogenic cirrhosis. Am. J. Gastroenterol, 99: 292-298.

14. Ikura Y. 2014. Transitions of histopathologic criteria for diagnosis of nonalcoholic fatty liver disease during the last three decades 12 (6): 894-900. World. J. Hepatol. 923-928.

15. Chaix A., Lin T., Le H.D., Chang M.W., Panda S. 2019. Time-restricted feeding prevents obesity and metabolicome in mice lacking a circadian clock. Cell metabolism. 29 (2): 303-319.

16. Käräjämäki A.J., Bloigu R., Kauma H., Kesäniemi Y.A., Koivurova O., Perkiömäki J., Huikuri H., Ukkola O. 2017. Non-alcoholic fatty liver disease with and without metabolic syndrome: different long-term outcomes. Metabolism. 66: 55-63. 
17. Kim D., Touros A., Kim W.R. 2018. Nonalcoholic fatty liver disease and metabolic syndrome. Clinics in liver disease. 22 (1): 133-140.

18. Lin Y.H., Liao Y.Y., Yeh C.K., K.C. Yang., Tsui P.H. 2018. Ultrasound entropy imaging of nonalcoholic fatty liver disease: Association with metabolic syndrome/entropy. 20 (12): 893.

19. Lucero D., Miksztowicz V., Gualano G., Longo C., Landeira G., Álvarez E., Zago V., Brites F., Berg G., Fassio E., Schreier L. 2017. Nonalcoholic fatty liver disease associated with metabolic syndrome: influence of liver fibrosis stages on characteristics of very low-density lipoproteins. Clinica chimicaacta. 473: 1-8.

20. Sebastiani G., Alshaalan R., Wong P., Rubino M., Salman A., Metrakos P., Deschenes M., Ghali P. 2015. Cardiovascular risk across the histological spectrum and the clinical manifestations of nonalcoholic fatty liver disease: An update. 2015 Jun 14; 21 (22): 6820-34. doi: 10.3748/wjg.v21.i22.6820. World. J. Gastroenterol. 102-107.

\section{References}

1. Denisov N.L., Grinevich V.B., Chernecova E.V., Kornouhova L.A., Vostrikova E.B., Chuprina S.V., Potapova I.V. 2017. Sovremennye koncepcii patogeneza ateroskleroticheskogo porazhenija sosudistoj stenki u pacientov s abdominal'nym ozhireniem i razlichnymi formami nealkogol'noj zhirovoj bolezni pecheni [Modern concepts of the pathogenesis of atherosclerotic lesions of the vascular wall in patients with abdominal obesity and various forms of non-alcoholic fatty liver disease]. Vestnik Nacional'nogo mediko-hirurgicheskogo Centra im. N.I. Pirogova. 1: 57-62.

2. Deomidova V.N., Petrova O.V.2013. Sravnitel'nyj analiz rezul'tatov jelastografii sdvigovoj volnoj i tranzientnojjelastografii $\mathrm{v}$ diagnostike diffuznyh zabolevanij pecheni [Comparative analysis of the results of shear wave elastography and transient elastography in the diagnosis of diffuse liver diseases]. Ul'trazvukovaja i funkcional'naja diagnostika. 5: 17-22.

3. Kilina O.Yu., Khanarin N.V., Ivanova S.N., Rossova N.A. 2015. Diagnosticheskiye vozmozhnosti ul'trazvukovogo issledovaniya $\mathrm{s}$ otsenkoy gepatorenal'nogo indeksa $\mathrm{v}$ diagnostike nealkogol'noy zhirovoy bolezni pecheni u bol'nykh metabolicheskim sindromom [Diagnostic capabilities of ultrasound examination with an assessment of hepatorenal index in the diagnosis of non-alcoholic fatty liver disease in patients with metabolic syndrome]. Vestnik KHGU im. N.F. Katanova. 12: 56-59.

4. Kosobjan E.P., Smirnova O.M. 2010. Sovremennye koncepcii patogeneza nealkogol'noj zhirovoj bolezni pecheni [Modern concepts of the pathogenesis of non-alcoholic fatty liver disease]. Saharnyj diabet. 1: 55-63.

5. Lelyuk V.G., Lelyuk S.E. 2003. Ul'trazvukovaya angiologiya [Ultrasound Angiology]. 2-ye izdaniye, dopiperer. Moskva. $336 \mathrm{~s}$.

6. Mit'kov V.V. 2011. Prakticheskoye rukovodstvo po ul'trazvukovoy diagnostike. Obshchaya ul'trazvukovaya diagnostika [A practical guide to ultrasound diagnostics. General ultrasound diagnostics]. Moskva. Vidar. $720 \mathrm{~s}$.

7. Nedogoda S.V., Sanina T.N., Chaljabi T.A., Barykina I.N., Coma V.V., Chumachek E.V., Salasjuk A.S., Hripaeva V.Ju., Smirnova V.O. 2014. Diagnostika i osobennosti lechenija nealkogol'noj zhirovoj bolezni pecheni u pacientov s metabolicheskim sindromom: vozmozhnosti kardiologicheskih $\mathrm{i}$ saharosnizhajushhih preparatov [Diagnostics and treatment features of non-alcoholic fatty liver disease in patients with metabolic syndrome: the possibilities of cardiological and hypoglycemic drugs]. MS. 11: $66-69$.

8. Tikhonov A.I., Khvorostinka V.N., Yefremova O.A., Yarnykh T.G., Pushkar S.N., Kotenko A.M. 1994. Sredstvo, obladayushcheye antioksioksidantnym i gepatozashchitnym svoystvami [An agent with antioxidant and hepatoprotective properties]. Patent naizobreteniye RU 2020946 C1, 15.10.1994. Zayavka № 4864094/14 ot 05.09.1990.

9. Khaybullina Z.R., Kosnikova I.V. 2014. Proyavleniya metabolicheskogo sindroma u bol'nykh mul'tifokal'nym aterosklerozom [Manifestations of metabolic syndrome in patients with multifocal atherosclerosis].Vestnik NGPU. 5 (21): 127-133.

10. Chesnokova L.V., Petrov I.M., Troshina I.A., Medvedeva I.V. 2014. Insulinorezistentnost', aterogennyye narusheniya i fibroz pecheni u bol'nykh s metabolicheskim sindromom [Insulin resistance, atherogenic disorders and liver fibrosis in patients with metabolic syndrome]. Ozhireniye i metabolizm. 2: $17-23$. 
11. Abenavoli L., Milic N., Di Renzo L., Preveden T., Medić-Stojanoska M., De Lorenzo A. 2016. Metabolic aspects of adult patients with nonalcoholic fatty liver disease. World. J. Gastroenterol. Aug 21; 22 (31): 7006-16.

12. Bhandarkar N.S., Brown L., Panchal S.K. 2019. Chlorogenic acid attenuates highcarbohydrate, high-fat diet-induced cardiovascular, liver, and metabolic changes in rats. Nutrition research. 62: 78-88.

13. Browning J.D., Kumar K.S., Saboorian M.H., Thiele D.L. 2004. Ethnic differences in the prevalence of cryptogenic cirrhosis. Am. J. Gastroenterol, 99: 292-298.

14. Ikura Y. 2014. Transitions of histopathologic criteria for diagnosis of nonalcoholic fatty liver disease during the last three decades 12 (6): 894-900. World. J. Hepatol. 923-928.

15. Chaix A., Lin T., Le H.D., Chang M.W., Panda S. 2019. Time-restricted feeding prevents obesity and metabolicome in mice lacking a circadian clock. Cell metabolism. 29 (2): 303-319.

16. Käräjämäki A.J., Bloigu R., Kauma H., Kesäniemi Y.A., Koivurova O., Perkiömäki J., Huikuri H., Ukkola O. 2017. Non-alcoholic fatty liver disease with and without metabolic syndrome: different long-term outcomes. Metabolism. 66: 55-63.

17. Kim D., Touros A., Kim W.R. 2018. Nonalcoholic fatty liver disease and metabolic syndrome. Clinics in liver disease. 22 (1): 133-140.

18. Lin Y.H., Liao Y.Y., Yeh C.K., K.C. Yang., Tsui P.H. 2018. Ultrasound entropy imaging of nonalcoholic fatty liver disease: Association with metabolic syndrome/entropy. 20 (12): 893.

19. Lucero D., Miksztowicz V., Gualano G., Longo C., Landeira G., Álvarez E., Zago V., Brites F., Berg G., Fassio E., Schreier L. 2017. Nonalcoholic fatty liver disease associated with metabolic syndrome: influence of liver fibrosis stages on characteristics of very low-density lipoproteins. Clinica chimicaacta. 473: 1-8.

20. Sebastiani G., Alshaalan R., Wong P., Rubino M., Salman A., Metrakos P., Deschenes M., Ghali P. 2015. Cardiovascular risk across the histological spectrum and the clinical manifestations of nonalcoholic fatty liver disease: An update. 2015 Jun 14; 21 (22): 6820-34. doi: 10.3748/wjg.v21.i22.6820. World J Gastroenterol. 102-107.

\section{ИНФОРМАЦИЯ ОБ АВТОРАХ}

Шишова Ирина Анатольевна, ассистент кафедры факультетской терапии медицинского института НИУ «БелГУ», врач УЗИ и функциональной диагностики Белгородского онкологического диспансера, г. Белгород, Россия

Ефремова Ольга Алексеевна, доктор медицинских наук, доцент, заведующая кафедрой факультетской терапии медицинского института НИУ «БелГУ», г. Белгород, Россия

Чернобай Павел Егорович, аспирант кафедры факультетской терапии медицинского института НИУ «БелГУ», г. Белгород, Россия

Абдулгани Шахноза, аспирант кафедры факультетской терапии медицинского института НИУ «БелГУ», г. Белгород, Россия

\section{INFORMATION ABOUT THE AUTHORS}

Irina A. Shishova, Assistant of the Department of Faculty Therapy, Medical Institute, Belgorod National Research University, Belgorod, Russia, doctor of ultrasound and functional diagnostics, Belgorod Oncological Dispensary, Belgorod, Russia

Olga A. Efremova, Doctor of Medical Sciences, Head of the Department of Faculty Therapy, Medical Institute, Belgorod National Research University, Belgorod, Russia

Pavel Ye. Chernobay, Postgraduate student of the Department of Faculty Therapy, Medical Institute, Belgorod National Research University, Belgorod, Russia

Abdulgani Shakhnoza, Postgraduate Student, Department of Faculty Therapy, Medical Institute, Belgorod National Research University, Belgorod, Russia 